\section{Mechanical Instabilities of Metals}

\author{
C.E. Bottani and G. Caglioti, Milan
}

(Istituto di Ingegneria Nucleare - CESNEF)

Temperature Changes during Deformation are Indices of Fundamental Processes

Traditionally, studies of the mechanical behaviour of solids are the prerogative of mechanical engineers or applied mathematicians. The former are generally more interested in deriving descriptions of the performance of structures rather than in understanding more basic aspects of the behaviour of condensed matter. Their analyses are usually based on the implicit assumption that mechanical transformations are indeed purely mechanical. Temperature is a parameter which enters as a static operating condition or, in certain instances, as determining the rates of processes that are thermally activated. Its role as a response to mechanically induced dynamic changes of state is generally ignored.

Applied mathematicians, on the other hand, tend to base their calculations on idealized models where discontinuities are introduced a priori, so that, for instance, a yielding surface in the stress tensor space is postulated rather than discovered as an inevitable result of a more fundamental model of material behaviour.

It is for the physicist to fill the gap between the basic micromechanisms taking place in the structure of real materials which contain defects and the dynamical processes of self-organisation occurring in materials which are driven off equilibrium. These processes are responsible for the strength of engineering structures. In such a task, the detailed function of temperature as a dynamic component cannot be ignored.

In order to define the state of a thermodynamic system in local equilibrium, or slightly removed from global equilibrium, the local temperature, i.e. the state variable conjugated to the local entropy in the local Helmholtz thermodynamic potential, is hierarchically as necessary as the strain tensor which is the set of state variables conjugated to the stress tensor.

Temperature and deformation turn out to be non-linearly coupled, and any local deformation transient produces a local temperature change. This coupling is in evidence even before the onset of plasticity, indeed, throughout the Hookean regime of elasticity.

\section{Thermoelastic Effect}

We begin with the simplest "mechanical" transformation immediately preceding the onset of plasticity which is essentially a thermoelastic deformation.

When a metallic sample is stretched by $\varepsilon_{Z}$ during a non isothermal tensile test, its relative cross-sectional area shrinks by $2 v \varepsilon_{Z}$ ( $v$ being the Poisson ratio) and the relative volume of the sample increases by

$$
\triangle V N=(1-2 v) \varepsilon_{Z}
$$

If the time needed to produce this volume increase is both

i) short with respect to the heat diffusion time along the sample axis and

ii) long enough to make the heat production by viscous effects negligible, the approximation of an isentropic process $(\triangle S=0)$ should apply.

The components of $\Delta S$ to be considered are:

1) the usual thermal entropy change, $C_{V} \Delta T / T_{o}$, where $C_{V}$ is the specific heat at constant volume and $T_{0}$ is the operating temperature;

2) the configurational entropy change associated with the deformation, i.e. $(\partial S / \partial V)_{T} \times(1-2 v) \varepsilon_{Z} V$. Using Maxwell's relation $(\partial S / \partial V)_{T}=(\partial p / \partial T)_{V}$ where $p$ is the pressure, and

$(\partial p / \partial T)_{V}=-(\partial p / \partial V) \times(\partial V / \partial T)$, one obtains $(\partial S / \partial V)_{T}=\beta / K_{T}$ in terms of the volume coefficient of thermal expansion, $\beta$, and the isothermal compressibility, $K_{T}$.

Isentropic conditions therefore lead to $\Delta T / T_{0}=-\gamma(1-2 v) \varepsilon_{z}$
Fig. 1 - A) Temperature, B) Stress, C) Strain against time for a standard slab of SAFC - 4 OR steel previously de. formed up to a total elongation of $1.4 \%$. Note the initial thermoelastic cooling, followed by plastic heating. where $\gamma$ is the Grüneisen parameter $=$ $\beta V / K_{T} C_{V}$.

During a tensile test performed under these conditions, the sample cools down while undergoing deformation in the elastic region. The amount of temperature drop is proportional to the deformation (which is presumed to occur homogeneously within the bulk of the sample) and depends on the Grüneisen parameter, which reflects the anharmonicity of the interatomic forces and gives a measure of the thermomechanical coupling. At ordinary temperatures, $\gamma$ lies between 1.5 and 2.5 for a wide range of metals.

The decrease in temperature we have noted, is opposed by heating effects that stem from the viscosity of the metal. As the applied stress increases, the initially sessile dislocations become more and more mobile and their motion within the sample generates an increasing amount of thermal energy. Consequently, in competition with the anharmonicity that gives rise to a stable thermoelastic cooling whose extent is determined by $\gamma$, a destabilizing mechanism with positive feedback is introduced through the incipient processes of plasticity. When the critical point is reached, the two mechanisms balance each other marking the boundary between the thermoelastic and thermoplastic regimes of deformation.

\section{Thermoelastic Limit Stress}

From a numerical stand point, a metallic sample deformed at room temperature, in practice cools down by no more than $1 \mathrm{~K}$ and so, in the past when temperature measurements, being based on thermocouples, were cumbersome, and the flow of heat from the clamps holding the sample could mask the effect, the change could be regarded as academic. The position has changed dramatically with the introduction of prompt semiconductor thermistors and it is now possible to measure reproducible changes of $0.001 \mathrm{~K}$ and less. This has permitted

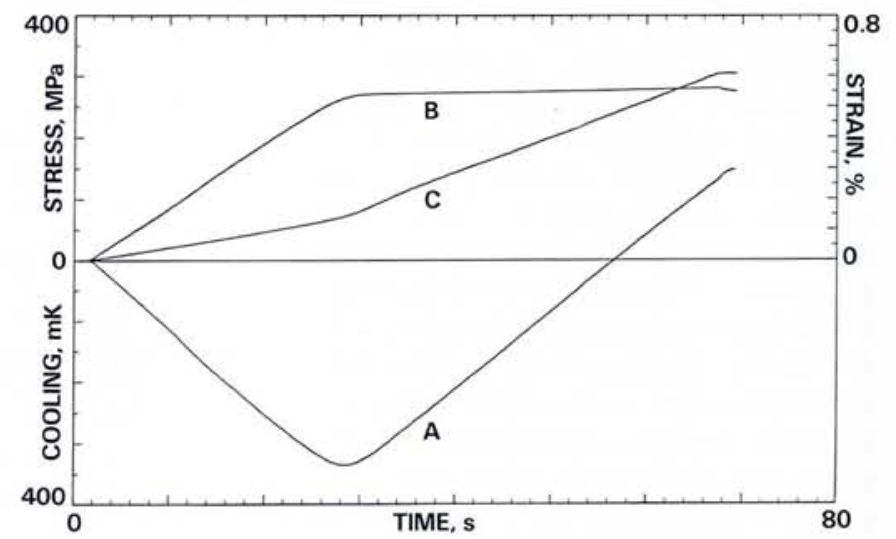




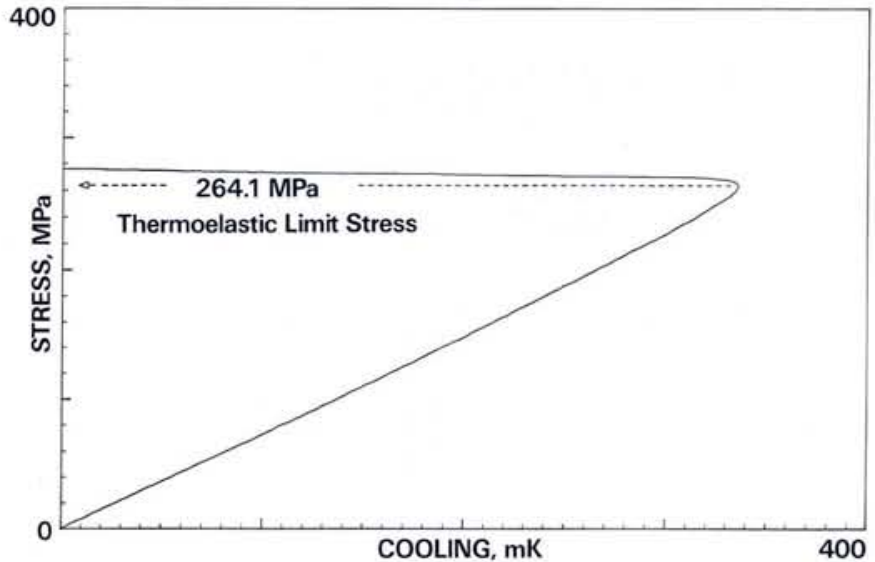

Fig. 2a) - By eliminating time between curves $A$ ) and $B$ ) of Fig. 1, one obtains the $T$ against $\sigma$ characteristic, showing a sudden change in the temperature trend at a thermoelastoplastic limit stress of $264.1 \mathrm{MPa}$.

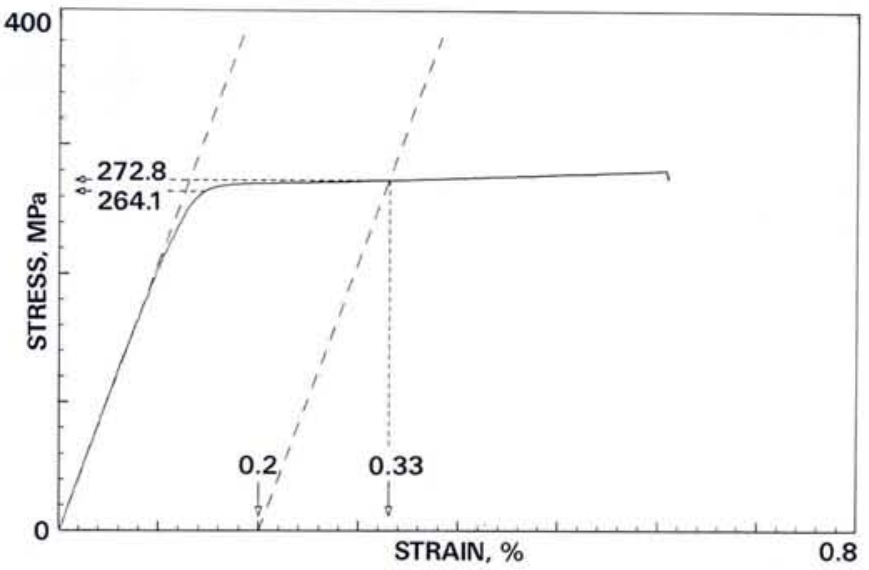

Fig. 2b) - By eliminating time between curves $B$ ) and C) of Fig. 1, one obtains the $\sigma$ against $\varepsilon$ characteristic. Due to work hardening, the two characteristic stresses differ by only about $10 \mathrm{MPa}$. In general, for an annealed steel sample, the two stresses differ by a few tens of $\mathrm{MPa}$. the production of characteristic stress curves such as that shown in Fig. 1.

The stress corresponding to the critical point marking the boundary between the Hooke-Kelvin thermoelastic and the thermoplastic regimes, we refer to as the thermoelastoplastic limit stress $\sigma_{\theta}$ or, simply, the thermoelastic limit (Fig. $2 a)$. The value of $\sigma_{\theta}$ turns out to be slightly smaller than the yield strength $\sigma_{0.2}$ currently derived from the stressstrain characteristic following standard procedures (Fig. 2b). However, its unambiguous definition could, we believe, eliminate the empirical safety (or ignorance) factors, commonly attached to $\sigma_{0.2}$ in structural design, even if only gradually. The temperature rise occurring at stress values immediately above $\sigma_{\theta}$ clearly marks the onset of the irreversible and irrecoverable behaviour of materials. Its adoption would allow the actual "yield" point of a material to be pinpointed with greater precision than can be obtained from $\sigma_{0.2}$.

\section{Theoretical Treatment}

Within the frame of the non-linear thermodynamics of irreversible phenomena, $\sigma_{\theta}$ could be interpreted as a bifurcation point: there, the linear thermodynamic branch becomes unstable while, under the control of the imposed deformation, a large temperature fluctuation grows as a component of the order parameter which "enslaves" the system and promotes its dynamical reorganization.

A model has been developed to explore the main features of this instability that is formally based on a system of two non-linear partial differential equations in the unknown temperature and deformation fields, expressing the balance of entropy and linear momentum within a sample undergoing a tensile test. The linearized equations associated with the above system determine the fluctuations of temperature and deformation around the time-dependent reference state, represented by the thermoelastic branch. The fluctuations are expanded in normal modes and the amplitudes of these temperature and deformation modes obey a system of linear equations. A linear stability analysis gives the threshold value of the control parameter at which the amplitude of the least damped, i.e. the fundamental mode, eventually diverges.

The above model refers to an ideally homogeneous anharmonic solid, where the defect contribution to the local thermodynamic functions is lumped in an effective phenomenological Grüneisen parameter. As such, the picture below for thermal emission based on the development of this model, should be given only a qualitative value.

\section{Thermal Emission}

The amplitude of the fundamental mode provides a measure of the fluctuations of both the temperature and the deformation. In our case, this mode is thermoacoustic with a frequency that is a complex number, the imaginary component of which equals that of the fundamental sound wave of the structure. In a thermoelastic anharmonic solid, thermal energy fluctuations and, consequently, temperature can propagate also in a wave-like fashion. The real component of the frequency represents the damping, and depends strongly on the size of the control parameter, i.e. the applied deformation. It is negative in the elastic region, but becomes positive across the thermoelastic critical point. Correspondingly, the order parameter exhibits a continuous transition from a zero mean value to a finite size: the originally incoherent phonon field tends to become partially coherent, as in the initial stage of the single mode continuous laser dynamic instability. In our opinion this is the origin of acoustic emission and, at the same time, of what one might call thermal emission.

Thermal emission during yielding has been detected and analyzed so far in hundreds of samples of metallic structural materials of which Figs. 1 and 2 represent only one instance. Here the knowledge of yield in the sample "as received" and after deep drawing into the shape of a car body was important.

\section{Plastic Regime}

In the plastic regime a fraction of the mechanical work done to deform plastically a solid body is unavoidably converted into heat through e.g. internal friction and the extensive irreversible motion of defects. At room temperature this fraction can reach about ninety per cent. Dissipation of this heat gives rise to a considerable increase in temperature which, in turn, modifies the mechanical susceptibility of the material itself (softening). Although at lower temperatures, because the metal is a poorer heat conductor, the fraction is more modest, a positive feedback can still originate in the deformation band via thermally activated processes of dislocation motion. In turn, plastic flow can exhibit higher order instabilities (e.g. the oscillatory behaviour of serrated yielding).

The physical index of dissipation is the entropy production (the specific entropy produced per unit time). In purely reactive diffusive systems as are to be found in chemical systems, this quantity controls the stability, whereas in a solid 
undergoing large deformations, while temperature stands as a relevant physical variable, the entropy production alone is no longer sufficient to provide a criterion for stability. Detailed models of cooperative defect kinetics must be worked out in addition.

Consequently, it seems advisable to extend the treatment proposed for the thermoelastic instability to other dynamic instabilities exhibited by materials under stress. We allude to creep, fatigue and fracture. Real-time monitoring of the temperature of metallic materials undergoing deformation might provide a means of detecting and highlighting internal mechanical processes responsible for the instabilities.

\section{FURTHER READING}

Proceedings of the International School of Physics "E. Fermi", Mechanical and Thermal Behaviour of Metallic Materials, Eds. G. Caglioti and A. Ferro Milone (North-Holland Pub. Co., Amsterdam) 1982.

\section{Solar Physics Section}

Following the elections which were concluded at the end of November, the Board of the Solar Physics Section is now composed as follows. Newly elected members are indicated by *

Ch.: M. Kuperus, Solar Observatory, Utrecht Sec.: R.W.P. McWhirter, Rutherford Appleton Lab., Chilton

* A. Benz, ETH, Zurich

* F. Chiuderi, University, Florence

* F.L. Deubner, University, Würtzburg

J.L. Leroy, Observatory, Bagnères de Bigorre

*W. Mattig, Institute for Solar Physics,

Freiburg

M.A. Pick, Observatory, Meudon

* E.R. Priest, University, St. Andrews

R.J. Rutten, Observatory, Utrecht

E.H. Schröter, Institute for Solar Physics, Freiburg

N. Weiss, University, Cambridge

To all our readers a Mery Christmas and a Happy and Peaceful 1984

\section{Research Associate Position in Experimental Nuclear Physics}

There is a vacancy for an experimental physicist to join the nuclear physics group as a Research Associate at the Daresbury Laboratory, an establishment of the Science and Engineering Research Council situated in the north Cheshire countryside.

The group is involved in a research programme on the Nuclear Structure Facility, a large tandem accelerator which has recently been completed and which currently is operating at up to $20 \mathrm{MV}$ on terminal.

Currently, the principal areas of study are: direct reactions with light and heavy ions, production of exotic nuclei far from stability, nuclei with very high angular momentum, nuclear breakup and fragmentation and studies of isotope shifts and hyperfine structure using laser-induced resonance fluorescence. Experimental equipment includes a Q3D magnetic spectrometer, advanced gamma-ray and neutron detection apparatus, and a $\mathrm{lm}$ diameter scattering chamber. An isotope separator with an associated dilution refrigerator and a beam line for laser studies is in an advanced commissioning stage, a recoil separator is due to begin initial testing by the end of the year, and a heavy-ion polarised source is under construction.

Applicants (male or female) should possess a $\mathrm{PhD}$ degree or expect to obtain one during 1983/early 1984 in nuclear physics.

An appointment will be made at a salary between $£ 6,985$ and $£ 10,686$ per annum depending on age, ability and experience. The post is available for a fixed term of three years and is superannuable.

CLOSING DATE: 28th February, 1984.

For further information please write or telephone Dr. J. S. Lilley on Warrington (0925) 65000 Ext. 558.

Application forms may be obtained from and should be returned quoting reference $\mathrm{DL} / 835$ to:

The Personnel Officer Science \& Engineering Research Council, Daresbury,

Warrington,

WA4 4AD

England.
Europhysics News is the official journal of the European Physical Society which comprises 29 National Societies, Academies and Group, over 3000 Individual Members and 66 Associate Members. Governing bodies of EPS are the General Meeting, Council and an elected Executive Committee responsible for detailed policy. EPS promotes the collaboration of physicists throughout Europe, orga out Europe, organising and harmonising conferences and publications, improving physics education, encouraging physics applications, awarding scholarships to sponsored schools in Erice. EPS publishes in addition to EN, Europhysics Conference Abstracts, E. Ed. News and, in collaboration with The Institute of Physics (UK). the European Journal of Physics. Individual Members receive EN free of charge (price to institutions: Sw F recelve EN froe of che of Phys

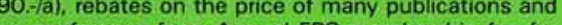
on conference fees. Annual EPS membership fee for Individual Members belonging to an EPS member society is: Sw.Fr. 40.; independent members: Sw.Fr. 120.-; Am. Phys. Soc. members: Sw.F. 50.- (\$ 25).
Editor: E.N. Shaw

Meetings Compilation: W.S. Newman Editorial Board:

K. Appert, A. Baratoff, B. Jacrot,

G.R. Macleod, A. Maeder, J. Muller

Editorial and Advertising Office at the EPS Secretariat

Address: EUROPEAN PHYSICAL SOCIETY P. O. Box 69 , CH- 1213 Petit-Lancy 2 Switzerland

Telephone: Geneva (22) 931130

Telex : $\mathbf{4 2 3} 455$ dema $\mathrm{ch}$

Cables: europhys genève

Printed by: Pfirter frères sa CH-1213 Petit-Lancy/Switzerland 